University of Nebraska - Lincoln

DigitalCommons@University of Nebraska - Lincoln

$9-2008$

Tissue and stage-specific juvenile hormone esterase (JHE) and epoxide hydrolase (JHEH) enzyme activities and Jhe transcript abundance in lines of the cricket Gryllus assimilis artificially selected for plasma JHE activity: Implications for JHE microevolution

\author{
Anurag Anand \\ University of Nebraska - Lincoln \\ Erica Crone \\ University of Nebraska - Lincoln \\ Anthony J. Zera \\ University of Nebraska - Lincoln, azera1@unl.edu
}

Follow this and additional works at: https://digitalcommons.unl.edu/bioscizera

Part of the Microbiology Commons

Anand, Anurag; Crone, Erica; and Zera, Anthony J., "Tissue and stage-specific juvenile hormone esterase (JHE) and epoxide hydrolase (JHEH) enzyme activities and Jhe transcript abundance in lines of the cricket Gryllus assimilis artificially selected for plasma JHE activity: Implications for JHE microevolution" (2008). Anthony Zera Publications. 29.

https://digitalcommons.unl.edu/bioscizera/29

This Article is brought to you for free and open access by the Papers in the Biological Sciences at DigitalCommons@University of Nebraska - Lincoln. It has been accepted for inclusion in Anthony Zera Publications by an authorized administrator of DigitalCommons@University of Nebraska - Lincoln. 
Published in Journal of Insect Physiology 54:9 (September 2008), pp. 1323-1331;

doi 10.1016/j.jinsphys.2008.06.006 Copyright (c) 2008 Published by Elsevier Ltd.

Used by permission. http://www.sciencedirect.com/science/journal/00221910

Submitted October 30, 2007; revised June 5, 2008; accepted June 6, 2008; published online June 26, 2008.

\title{
Tissue and stage-specific juvenile hormone esterase (JHE) and epoxide hydrolase (JHEH) enzyme activities and Jhe transcript abundance in lines of the cricket Gryllus assimilis artificially selected for plasma JHE activity: Implications for JHE microevolution
}

\author{
Anurag Anand, Erica J. Crone, and Anthony J. Zera \\ School of Biological Sciences, University of Nebraska-Lincoln, Lincoln, NE 68588-0118, USA \\ Corresponding author-A. J. Zera, tel 402 472-2768, fax 402 472-2083, email azera1@unl.edu
}

E. J. Crone's current address is: Office of the Gene Technology Regulator, MDP 54 GPO Box 9848, Canberra ACT 2601, Australia.

\begin{abstract}
Fat body and midgut juvenile hormone esterase (JHE) and juvenile hormone epoxide hydrolase (JHEH) specific activities, and plasma JHE activity, were measured throughout the last stadium in two pairs (blocks) of lines of the cricket Gryllus assimilis, each pair of which had been artificially selected for high- or low-plasma JHE activity. Highly significant differences were observed between high- and low-activity lines of each block on most days for fat body JHE, and on one day for midgut JHE activity. In each block, line differences in developmental profiles for fat body JHE activity paralleled line differences in plasma JHE activity during the early-mid stadium, but not during the latter part of the stadium. The developmental profile of midgut JHE activity differed from that of plasma and fat body JHE activity, exhibiting peaks during the early and latter parts of the stadium. Midgut and fat body JHEH activities exhibited a mid-stadium peak in all lines, but activities were very similar in all lines. Fat body JHE appears to be a more significant contributor to plasma JHE than is midgut JHE. During the middle of the last stadium (day 4), Jhe transcript abundance was significantly higher in fat body or midgut of highvs. low-JHE-activity lines. Jhe transcript abundance was positively correlated with JHE enzyme activity in either fat body or midgut, and with plasma JHE activity. Natural populations of G. assimilis harbor genetic variation for Jhe gene expression which appears to contribute to genetic variation in JHE specific activity in fat body and midgut. These genes appear to have been the targets of artificial selection that resulted in lines that differ dramatically in high- or low-plasma JHE activity. These genes appear to have little, if any, pleiotropic effects on JHEH specific activity.
\end{abstract}

Keywords: juvenile hormone esterase, JHE, juvenile hormone epoxide hydrolase, JHEH, juvenile hormone gene expression, artificial selection, evolution, microevolution

\section{Introduction}

The origin of endocrine adaptations is a key issue in evolutionary endocrinology. Endocrine adaptations originate with natural selection differentially favoring genetically-encoded hormonal variants (e.g. for blood titers, receptor characteristics, activities of hormone-regulating enzymes, etc.) segregating within populations of species. However, relatively little is known about the characteristics of this intraspecific endocrine variation, which, in turn, limits our understanding of the mechanisms underlying endocrine microevolution (Zera, 2006; Zera et al., 2007).

Juvenile hormone esterase (JHE) activity in species of Gryllus crickets has been intensively studied from an evolutionary-genetic perspective (reviewed in Zera, 2006; and Zera et al., 2007). JHE hydrolyzes the key developmental and repro- ductive hormone, juvenile hormone (JH), and partially regulates its titer (Hammock, 1985; Roe and Venkatesh, 1990; Goodman and Granger, 2005). Genetically-based variation in plasma (blood) JHE activity has been documented in recently-founded laboratory populations of several cricket species (Zera and Tiebel, 1989; Zera and Zhang, 1995; Roff et al., 1997; Zera and Huang, 1999; Zera, 2006). In wing-polymorphic species, elevated plasma JHE activity during the last nymphal stadium is strongly associated genetically with the subsequent production of the flight-capable (long-winged) as opposed to the flightless (short-winged)/reproductive morph (Zera and Tiebel, 1989; Roff et al., 1997; Zera and Huang, 1999). This strong association implicates selection on genetic variation for JHE activity as an important component of the evolution of flightlessness in Gryllus (reviewed in Zera, 2004; Zera, 2006; Zera et al., 2007). 
Thus far, our functional-genetic studies of JHE variation have focused almost exclusively on the characteristics, correlations and functional consequences of JHE activity in the plasma of artificially-selected lines of three Gryllus species (reviewed in Zera, 2006; Zera et al., 2007; Crone et al., 2007; see Section 4). Results to date collectively indicate that genetic alterations in JHE activity in the plasma during the last nymphal stadium (1) give rise to altered JH metabolism and expression of JH-regulated traits, and (2) likely result from variation in some aspect of JHE gene regulation rather than JHE allozymes that differ kinetically due to variation in primary amino acid sequence or posttranslational modification.

Plasma JHE originates in non-hemolymph tissues such as the fat body, and is secreted into the hemolymph (Hammock, 1985; Roe and Venkatesh, 1990; Goodman and Granger, 2005). Thus, it is essential to investigate JHE in various non-hemolymph tissues, to understand both the causes of hemolymph JHE activity difference between genetic stocks, as well as to understand the function of JHE in non-hemolymph tissues. In the present study, we measured specific activities of JHE, as well as JH-epoxide hydrolase (JHEH), the other main JH-degrading enzyme, in fat body and midgut, throughout the last nymphal stadium in genetic stocks of Gryllus assimilis artificially selected for high- or low-plasma JHE activity. These tissues, especially fat body, were chosen since they exhibit high JHE specific activity and are likely major contributors to plasma JHE activity (Wing et al., 1981; Hammock, 1985; Roe and Venkatesh, 1990; Jesudason et al., 1992). In addition, using a recently obtained the cDNA sequence from G. assimilis (Crone et al., 2007), we measured Jhe transcript abundance in both fat body and midgut. The main goal was to begin to directly assess the extent to which genetic variation for Jhe gene expression is associated with and contributes to genetic variation in plasma JHE activity in G. assimilis.

\section{Materials and methods}

\subsection{Stocks}

Studies were conducted on stocks of G. assimilis that had been artificially selected for high- or low-plasma JHE activity. These are the same lines in which aspects of JHE activity have been extensively characterized previously (Zera and Zhang, 1995; Zera et al., 1996; Zera et al., 1998; reviewed in Zera, 2006). Stocks had been maintained under the same environmental conditions as those under which the original artificial selection studies had been conducted (e.g., $28^{\circ} \mathrm{C}$; 16 light: 8 dark photoregime; cricket density and diet as reported in Zera and Zhang (1995)). Stocks had been periodically re-selected for high- or low-plasma JHE activity to maintain differences between lines in the activity of this enzyme. The original artificial selection study consisted of three independent selection trials (Blocks, Bks), each of which contained a line selected for high-plasma JHE activity, a line selected for low activity, and an unselected control (Zera and Zhang, 1995; Zera, 2006). In the present study, high- and low-selected lines of two of the three blocks (Bks 1 and 3) were studied. The duration of the last nymphal stadium ranged from 7 to 9 days with an average of 8 days.

\subsection{Tissues sampled and preparation}

Plasma JHE activity, and fat body and midgut JHE and JHEH specific activities were measured throughout the last nymphal stadium in JHE-selected lines. As mentioned above (Section 1; also see Section 4), fat body and midgut were studied because they exhibit high activities of these enzymes in several insect species, and thus were viewed as the most likely source of he- molymph JHE activity in G. assimilis. JHE transcript abundance was measured in fat body and midgut on day 4 of the last stadium, a day just prior to (midgut) or on which (fat body) JHE specific activity differs considerably between selected lines (see Section 3). Plasma was collected and stored as described previously (Zera and Huang, 1999). Briefly, $2 \mu \mathrm{L}$ hemolymph, obtained from cuts in the cerci, were diluted 10 -fold with $0.1 \mathrm{M}$ potassium phosphate buffer, $\mathrm{pH}$ 7.1. Solutions were centrifuged $(14,000 \times g, 10 \mathrm{~min})$, and supernatants (plasma) stored at $-86^{\circ} \mathrm{C}$ until assayed. Midgut and fat bodies were removed from crickets, tissues were rinsed in saline $(0.15 \mathrm{M} \mathrm{NaCl})$, blotted, transferred to preweighed eppendorf tubes, snap frozen in liquid nitrogen, and stored at $-86^{\circ} \mathrm{C}$ until use. Background experiments demonstrated no loss in enzyme activity in frozen whole tissues or in diluted plasma during the period of storage (up to several months).

\subsection{Enzyme assays}

Plasma JHE activity was assayed using the radiochemical partition assay of Hammock and Sparks (1977), slightly modified for use in Gryllus (Zera and Huang, 1999). Fat body and midgut JHE and JHEH activities were simultaneously measured using a thinlayer chromatographic (TLC) assay similar to that described in Hammock and Sparks (1977). The partition assay of Share and Roe (1988), which also has been used to simultaneously measure JHE and JHEH activities, gave inconsistent results in background studies and was not used. Fat body and midgut samples were homogenized in $0.1 \mathrm{M}$ potassium phosphate buffer, $\mathrm{pH} 7.1$, centrifuged for $15 \mathrm{~min}$ at $7000 \times \mathrm{g}$ at $4{ }^{\circ} \mathrm{C}$, and the supernatant was assayed immediately. This gentle centrifugation step increased the duration of linearity of the JHE/JHEH assays, possibly by removing cellular debris that binds added $\mathrm{JH}$, while not removing any JHE or JHEH activity. The pellet, after having been washed twice with homogenization buffer and suspended in homogenization buffer, exhibited $<3 \%$ of JHE or JHEH activity found in the supernatant. All samples were collected during a $4 \mathrm{~h}$ period between $4 \mathrm{~h}$ after lights on and $8 \mathrm{~h}$ before lights off. No significant diurnal change in JHE activity was observed during this time.

For the TLC assay, $20 \mu \mathrm{L}$ of suitably diluted tissue homogenate was added to $178 \mu \mathrm{L}$ of $0.1 \mathrm{M}$ potassium phosphate buffer, $\mathrm{pH}$ 7.1. The buffer contained $2 \mu \mathrm{L}$ of an ethanolic solution of radio-labeled racemic $\left[10-{ }^{3} \mathrm{H}\right]$-juvenile hormone III (10-20 Ci/ mmol, 370-740 GBq/mmol; PerkinElmer Life and Analytical Sciences) and racemic unlabelled JHE III (Sigma-Aldrich, Inc.) resulting in a final conc. of JH III of $5 \mu \mathrm{M}$ and containing 10,000 DPM per assay. Test tubes containing assay solution had been placed in an ice bath for a few minutes before addition of the tissue homogenate. Tubes were transferred from the ice bath to a $30{ }^{\circ} \mathrm{C}$ water bath, shaken for $30 \mathrm{~s}$ and incubated for $30 \mathrm{~min}$. Reactions were stopped by placing racks of tubes back into the ice bath. To each tube was added $1.5 \mu \mathrm{L}$ of $6 \mathrm{M} \mathrm{HCl}$ to lower the $\mathrm{pH}$ to 6.0 to aid in the extraction of JH acid. Ethyl acetate ( $200 \mu \mathrm{L})$ was added to each tube, tubes were vortexed for $1 \mathrm{~min}$, and centrifuged for $5 \mathrm{~min}$ at $4{ }^{\circ} \mathrm{C}$ at $5000 \mathrm{rpm}$. The organic (top) phase was removed using a pulled Pasteur pipette. The extraction step was repeated, the organic phases were combined, and solvent was removed under a stream of nitrogen. Diethyl ether $(100 \mu \mathrm{L})$ was added to each tube, which was briefly vortexed; solutions were then spotted onto plastic-backed silica gel TLC plates with $254 \mathrm{~nm}$ fluorescent indicator (Sigma-Aldrich, Inc.) that had been washed in methanol. JH, JH-diol, JH-acid, and JH-acid-diol standards were added to one lane of the plate. Plates were developed using the following solvent system: hexane:ethyl acetate: acetic acid (55:45:2 drops in $100 \mathrm{~mL}$ ). Spots corresponding to $\mathrm{JH}$, 
JH-acid, JH-diol, and JH-acid-diol were cut out, placed in vials containing Ready Safe cocktail (Beckman Coulter, Inc.) and radioactivity (DPM) quantified using an LKB liquid scintillation counter. Protein concentration was determined for the fat body and midgut homogenates using the Bradford protein assay (BioRad laboratories, Hercules, CA).

Background experiments were conducted to determine the amount of diluted plasma, fat body homogenate, or midgut homogenate required to produce rates that were linear with respect to time or tissue concentration for each of the metabolites of JHE/JHEH (JH-acid, JH-diol and JH-acid-diol). Assays were always run within these limits. Background experiments also demonstrated that it was unnecessary to add unlabelled $\mathrm{JH}$ or $\mathrm{JH}$ metabolites ("carriers") during extraction, which significantly reduced the cost of the large number of assays conducted. Addition of unlabelled JH and JH-acid during the ethyl acetate extraction improved the total recovery of radio-labeled $\mathrm{JH}$ and metabolites (75\% vs. $50 \%$ without cold carriers). However, the relative amount of $\mathrm{JH}$ and its metabolites extracted was the same whether carriers were used or not. Because reaction rates are estimated in this assay from the relative proportion of $\mathrm{JH}$ and its metabolites, reaction rates estimated with or without cold carriers did not differ significantly. In the JHE/JHEH assay of fat body and midgut homogenates, only a small amount $(1-2 \%)$ of $\mathrm{JH}$ acid-diol was produced (2-3\% of total $\mathrm{JH}$ metabolites produced), which was ignored in computation of JHE and JHEH activities.

$\mathrm{JH}, \mathrm{JH}$-acid, JH-diol, and JH-acid-diol standards were produced by incubating $300 \mu \mathrm{g}$ racemic JH III in $1 \mathrm{~mL}$ of potassium phosphate buffer, $\mathrm{pH} 7.1$, containing diluted plasma and fat body homogenate from a day 3-5 last stadium G. assimilis from the high-activity line. The solution was incubated for $5 \mathrm{~h}$ at $30^{\circ} \mathrm{C}$, and metabolites were extracted as in the JHE/JHEH TLC radiochemical assays described above. Rf values for $\mathrm{JH}$, JH-acid, $\mathrm{JH}$-diol, JH-acid-diol were $0.64,0.50,0.19,0.1$, respectively, and were similar to values obtained for chemically-synthesized metabolies (Zera and Tiebel, 1989).

\subsection{JHE transcript abundance in fat body and midgut. RNA isolation}

Total RNA was isolated from individual tissues using Trizol Reagent (Invitrogen, Carlsbad, CA) following manufacture's recommendations. RNA was resuspended in RNase free water and stored at $-20^{\circ} \mathrm{C} .5 \mu \mathrm{g}$ of total RNA was treated with 5 units of RNase free DNase 1 (Promega, Madison, WI) and reverse transcribed with 200 units of SuperScript II Reverse Transcriptase (Invitrogen, Carlsbad, CA) using an oligo $\mathrm{dT}_{18}$ oligonucleotide at $42{ }^{\circ} \mathrm{C}$ for $1 \mathrm{~h}$ in a final reaction volume of $40 \mu \mathrm{L}$.

\subsection{Amplification of $G$. assimilis actin sequence (internal control)}

The abundance of actin transcript was used as an internal control to standardize JHE transcript abundance in midgut and fat body of $G$. assimilis. Because the actin sequence had not yet been obtained from G. assimilis, it was amplified as follows: A consensus sequence generated by a ClustalW alignment of the Ovis aries, Homo sapiens, Cavia porcellus, Rattus norvegicus, and Drosophila melanogaster $\beta$-actin amino acid sequences was used to design oligonucleotide primers to amplify a $959 \mathrm{bp}$ internal region of the G. assimilis actin cDNA. $1 \mu \mathrm{L}$ of cDNA was used as a template in $1 \times \mathrm{PCR}$ buffer, $1 \mathrm{mM} \mathrm{MgCl}$, $0.2 \mathrm{mM}$ each dNTP, 5 units Taq DNA polymerase (Invitrogen, Carlsbad, CA) and the primers actinF1 5'GCGTGCTGTTTTCCCGTCCA3' and actinR1 5'GTCGTATTCCTGTTTGGAGA3' at a final concentration of $0.2 \mu \mathrm{M}$ each. PCR was carried out with an initial DNA denaturing step of $94^{\circ} \mathrm{C}$ for $2 \mathrm{~min}$, then 30 cycles of denaturation at $94^{\circ} \mathrm{C}$ for $30 \mathrm{~s}$, annealing at $53^{\circ} \mathrm{C}$ for $30 \mathrm{~s}$ followed by an extension step of $90 \mathrm{~s}$ at $72{ }^{\circ} \mathrm{C}$ and a final extension step of $7 \mathrm{~min}$ at $72{ }^{\circ} \mathrm{C} .1 \mu \mathrm{L}$ of a $1 / 100$ dilution of the previous reaction was used as template in a hemi-nested PCR with the same components with the exception that the antisense primer was substituted with actinR2 5'GAGATCCACATCTGCTGGAA3', and the annealing temperature was $55^{\circ} \mathrm{C}$. The hemi-nested amplicon was cloned into the pGem-T Easy vector (Promega, Madison, WI) and sequenced using BigDye terminator chemistry (Applied Biosystems, Foster City, CA). The actin nucleotide sequence obtained (available on request) was 959 bp in length and was $96 \%$ identical to Gryllus bimaculatus actin nucleotide sequence.

\subsection{Real time-polymerase chain reaction (real time-PCR) of JHE and} actin

Primer Express software (Applied Biosystems, Foster City, CA) was used to design the following taqman probes and primers to amplify the G. assimilis Jhe and actin cDNA based on the G. assimilis actin sequence obtained in the present study (see above), and the G. assimilis The sequence reported in Crone et al. (2007): ACTprobe: 5'6-FAM-CCTGTTCTCCTGACTGAAGCCCCTCTG-TAMARA3'; JHE probe: 5'6-FAM-CAGCCGCACGTCTCCCTCGA-TAMARA3'; Actin primers: 5'GAGTTGCCCCTGAGGAGCA3' (actin170 U) and 5'TTTCCCTGTTGGCTTTAGGGT3' (actin238L); Jhe primers: 5'AATCTCAGACTTCTACTTCGGCAAC3' (Jhe1098U) and 5'ATGAACGGCTGCGGCTT3' (Jhe1163L). Real time-PCR reactions were performed in a final volume of $50 \mu \mathrm{L}$ using TaqMan Universal PCR Master Mix (Applied Biosystems, Foster City, CA). The final concentration of each primer was $900 \mathrm{nM}$ and the final concentration of each probe in the reaction was $250 \mathrm{nM}$. Reactions were performed using 60 cycles of the manufacturer set cycling conditions on an ABI7700 real-time PCR machine (Applied Biosystems, Foster City, CA). Transcript abundance value of each individual was the mean of three replicates. Separate reactions were performed for the and actin, and the Jhe transcript measurements were normalized using actin as the standard. Where the standard deviation of normalized replicate samples was greater than 0.3 the individual was not included in the statistical analysis. To ensure that sample plates could be accurately compared to each other, standard samples were included on each plate. The threshold value for each plate was then set at a consistent value for the standard samples between plates and an allowed standard deviation of no more than 0.3 was maintained between plates. Standard curves were generated using a serial dilution of a mixture of cDNA prepared from Blocks 1 and 3 day- 4 samples. Standard PCR using the real time PCR primers yielded amplification products of expected size for both the and actin and preliminary real-time runs yielded expected amplification plots.

\subsection{Statistical analyses}

Analyses of differences between traits involving more than one variable (e.g., differences in transcript abundance between Line and Block) were performed using ANOVA (Sokal and Rohlf, 1989), while comparisons between pairs of means for a single variable (JHE or JHEH activity differences between high- and low-activity lines on a particular day of development) were performed using $t$-tests (Sokal and Rohlf, 1989). Associations between JHE and JHEH activity or between the transcript abundance and JHE activity were assessed by computing Pearson correlations [Spearman (non-parametric) correlations gave virtually identical results (data not shown)]. In the former case, all variables (i.e., enzyme activities; see Table 1) were tested against each other and the significance of the correlation coefficients was determined by taking into account the number of independent variables tested (a posteriori tests) using Table Y of Sokal and Rohlf (1969). Sample sizes and hence statistical power was much lower (about 10-fold) for corre- 
lations involving transcript abundance. Hence we only computed selected correlations that tested specific a priori hypotheses of interest, which did not require adjusting $P$-values for multiple variables. ANOVAs and correlation coefficients were computed using Systat 8.0, while $t$-tests were computed using Prism 4.0.

Table 1. Correlations between plasma, fat body, and midgut JHE and JHEH specific activities

\begin{tabular}{llllll}
\hline First variable & \multicolumn{2}{c}{ Second variable } & & & \\
& FB-JHE & FB-JHEH & PL-JHE & MG-JHE & MG-JHEH \\
\hline FB-JHE & & - & & & \\
& & & & & \\
FB-JHEH & $0.207 \mathrm{~ns}$ & - & & & \\
PL-JHE & $0.670^{* * *}$ & $0.107 \mathrm{~ns}$ & - & & \\
MG-JHE & $0.315^{* *}$ & $0.062 \mathrm{~ns}$ & $0.253^{*}$ & - & \\
MG-JHEH & $0.156 \mathrm{~ns}$ & $-0.017 \mathrm{~ns}$ & $0.369^{* *}$ & $0.397^{* *}$ & - \\
\hline
\end{tabular}

${ }^{* \star *} P<0.005 ;{ }^{* *} P<0.01,{ }^{*} P<0.05$, ns: non-significant $(P>0.05)$; correlations were based on 207 individual crickets sampled in the four artificially-selected lines on various days of the last stadium (data in Figure 1 and Figure 2; see Section 2 for additional details of correlational analyses). FB: fat body, MG: midgut, PL: plasma; JHE and JHEH: juvenile hormone esterase or juvenile hormone epoxide hydrolase specific activities, respectively.

\section{Results}

3.1. Enzyme activity profiles in various tissues and days of the last stadium

Activities of plasma JHE, specific activities of fat body and midgut JHE, and specific activities of fat body and midgut JHEH were measured in approximately 220 individual crickets from two pairs (two blocks) of lines. Each pair consisted of a line that had been artificially selected for high- or low-plasma JHE activity. Developmental profiles of plasma JHE activity, observed in the present study (Figure 1), were similar to profiles observed in previous studies (Zera and Zhang, 1995; Zera, 2006). Activities rose from near zero values at the beginning of the last stadium to a mid-stadium (day 4) peak of about $75 \mathrm{nmol} \mathrm{JH-acid/min/}$ $\mathrm{mL}$ plasma, and dropped again during the latter part of the stadium. Consistent differences were observed in JHE activity between high- and low-activity selected lines in each of the two blocks: in each case, peak JHE activities were about 7-fold higher in the high- vs. low-activity lines (results of $t$-tests are contained in the legend of Figure 1).

JHE activity in fat body was also significantly higher (5-10fold at peak) in crickets from the high-selected vs. low-selected
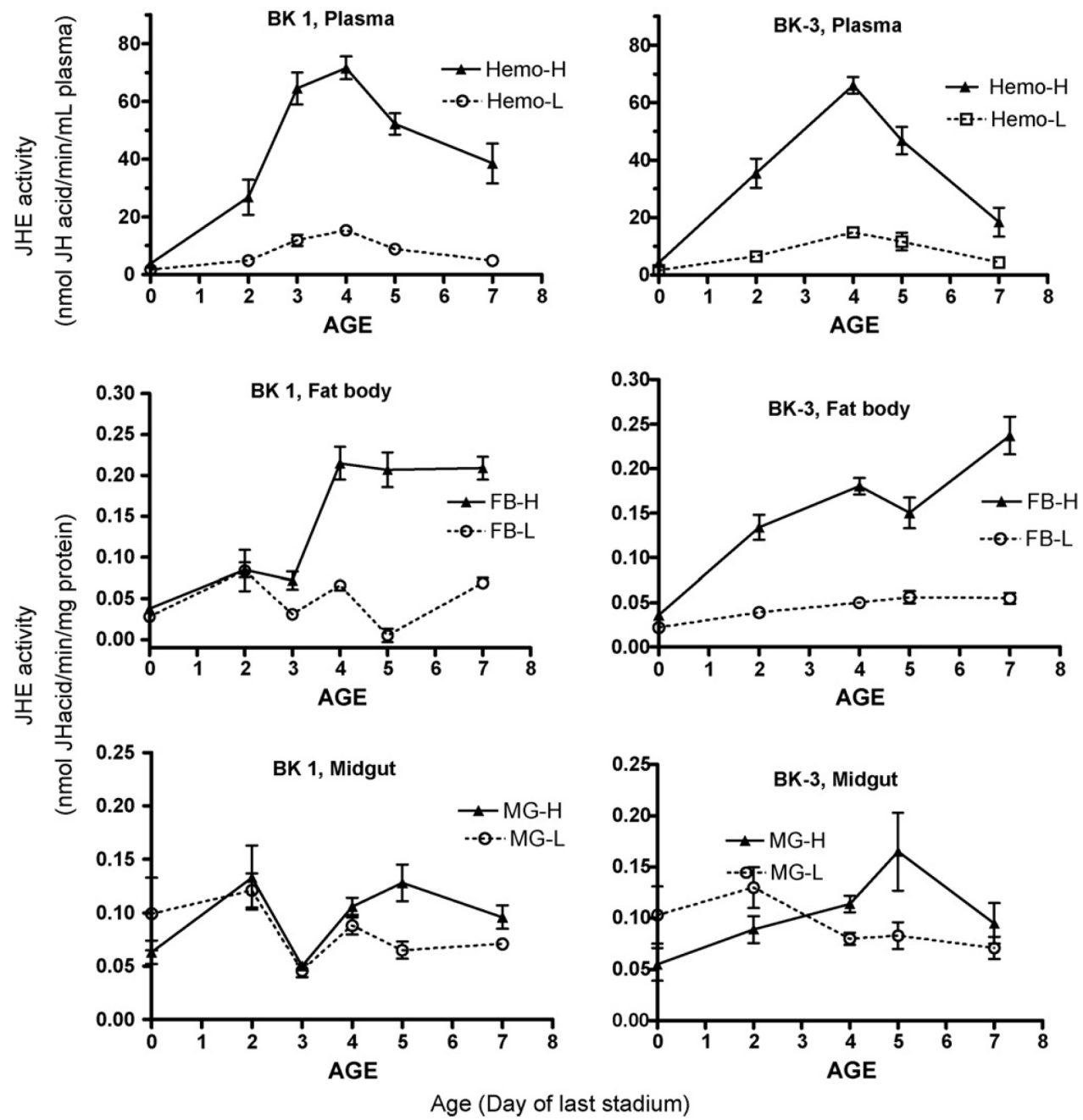

Figure 1. JHE activities (means \pm S.E.M.) in plasma, fat body, and midgut of lines of G. assimilis artificially selected for high (filled symbols, solid lines) or low (open circles, dashed lines) plasma JHE activity. Bk (block) refers to independent selection trial (see Section 2). Means were based on assays of 3-9 individuals (median $=4$ ) on days $0-3$ and 7-22 (median $=8$ ) individuals on days 4-7. JHE activities differed between high- and lowselected lines in the following cases for Bk-1 ( $P<0.05$; two-tailed $t$-test with unequal variances): plasma: all days except day 0 ; fat body: days 4,5 , and 7; midgut: day 5. Corresponding results for Bk-3: plasma: all days; fat body: all days; midgut: day 4. 

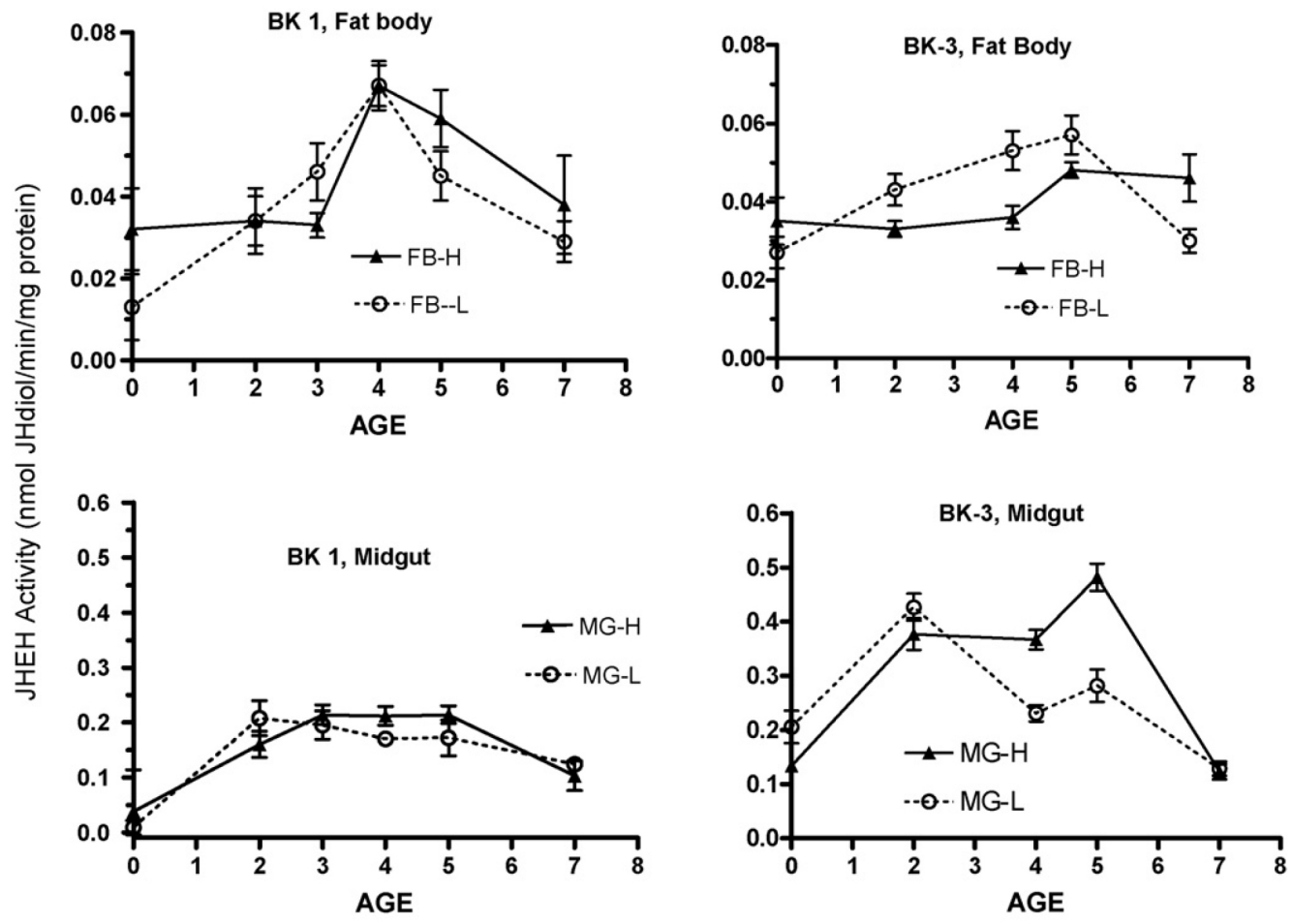

Age (Days of last stadium)

Figure 2. Juvenile hormone epoxide hydrolase specific activity in fat body and midgut of lines of G. assimilis artificially selected for high- or lowplasma JHE activity. See Figure 1 for explanation of symbols. Means were based on assays of 3-9 individuals (median = 4) on days 0-3 and 7-22 (median $=8$ ) on days 4-7. JHEH activities differed between high- and low-selected lines on the following days $(P<0.05$, two-tailed $t$-test with unequal variances): Bk-1, fat body: no day; Bk-1, midgut: day 4; Bk-3, fat body: days 2, 4, and 7; BK-3 midgut: days 4 and 5.

lines during the mid-latter part of the stadium (Bk-1) or during most of the stadium (Bk-3) (Figure 1; results of individual $t$-tests are in figure legend). JHE activity differences between high and low lines in fat body roughly paralleled activity differences in the plasma during most, but not all, portions of the last stadium, and some differences between the blocks were observed. Like plasma JHE activity, JHE activity in the fat body of crickets from the high-selected lines rose from its lowest value at the beginning of the stadium to a mid-stadium (day 4) peak (Bk-1) or near peak (Bk-3). Unlike plasma JHE activity, fat body JHE activity in the high-activity lines did not drop from the mid-stadium peak during the latter part of the of the stadium; activities either remained on a plateau (Bk-1), or rose slightly higher near the end of the stadium (Bk-3). Only slight changes in fat body JHE specific activity were observed in the low-activity lines during the last stadium, similar to the situation for plasma JHE activity.

The magnitude of peak JHE activity in the midgut was about half that of JHE activity in the fat body (Figure 1), and developmental profiles in the midgut differed from those in the fat body. First, rather than exhibiting a mid-stadium (day 4) peak, JHE activities in the midgut exhibited peaks early (day 2) and slightly later (day 5) in the stadium (Bk-1), or only later in the stadium (Bk-3). Second, JHE activities in the midgut differed to a lesser degree, and mainly on day 5, between the high-activity and lowactivity lines (results of $t$-tests are in legend of Figure 1).

JHEH activities in the midgut were much higher (ca. 5-fold) than JHEH activities in the fat body (Figure 2). Developmental profiles were roughly similar to plasma JHE activity (except for activities in the high-selected line of Bk-3). In general, activities rose to a broad mid-stadium peak (days 2-5) and fell near the end of the stadium. Virtually identical JHEH activities in the midgut and fat body were observed between high-activity and low-activity stocks of Bk-1 (results of $t$-tests are in the legend of Figure 2). In Bk-3, JHEH activity was consistently higher in the midgut of the high- vs. low-activity line during the mid-latter part of the stadium, while the low-activity line exhibited higher JHEH activity than the high-activity line during the mid-stadium in the fat body.
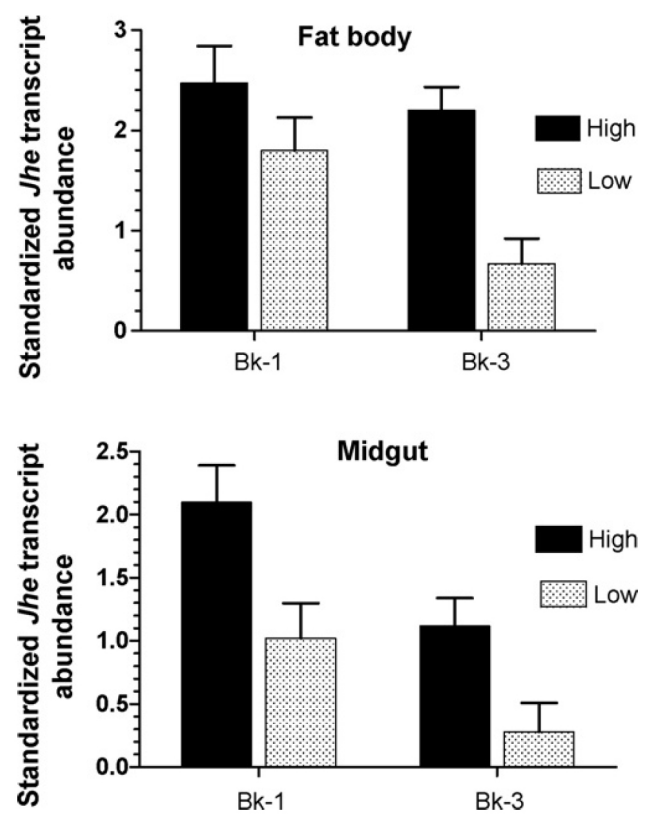

Figure 3. Standardized fat body, or midgut JHE transcript abundance in lines selected for high- or low-plasma JHE activity. Bk refers to independent selection trial (see Section 2). Values are means \pm S.E.M. based on 9-10 (Bk-1) or 4-6 (Bk-3) individuals. Each individual transcript abundance value, in turn, was the average of 3 replicates measured by real-time PCR (see Section 2). 
Table 2. Correlations $(r)$ between JHE transcript abundance and JHE activity in fat body, midgut or plasma in lines selected for high- or lowplasma JHE activity

\begin{tabular}{llll}
\hline First variable & & Second variable & $r$ \\
\hline FB-JHE-trans & vs. & FB-JHE-activity & $0.502^{*}$ \\
FB-JHE-trans & vs. & MG-JHE-trans & $0.597^{* *}$ \\
FB-JHE-trans & vs. & PL-JHE activity & $0.502^{*}$ \\
MG-JHE-trans & vs. & MG-JHE activity & $0.504^{*}$ \\
MG-JHE-trans & vs. & PL-JHE activity & $0.465^{*}$ \\
\hline
\end{tabular}

FB: fat body, MG: midgut, PL: plasma, JHE: juvenile hormone esterase, trans: transcript abundance (mRNA concentration), activity: enzyme specific activity; ${ }^{*} P<0.05$ and ${ }^{* *} P<0.01$ (Pearson correlations based on 28 crickets analyzed on day 4 of the last nymphal stadium in high and low lines of Bk-1 and Bk-3). These correlations were measured on the same individuals on which correlations reported in Table 1 were measured. Spearman (nonparametric) correlation coefficients were virtually identical to Pearson correlation coefficients and gave identical $P$ values.

\subsection{Correlations between JHE and JHEH activities in various body compartments}

Plasma JHE activity was most strongly and positively correlated with fat body JHE specific activity, and to a lesser degree with midgut JHE specific activity (Table 1). Fat body and midgut JHE activities were also positively correlated with each other. Midgut JHEH was correlated with plasma and midgut JHE, while fat body JHEH activity was not correlated with any measured variable.

\subsection{JHE transcript abundance in fat body and midgut}

The transcript abundance in fat body was measured on day 4 , the midpoint of the last nymphal stadium (Figure 3), a time in development just prior to (midgut) or when JHE activity peaks in fat body and plasma. Fat body transcript abundance, averaged over the 2 blocks, was 1.9-fold higher in the lines selected for high- vs. low-plasma JHE activity (ANOVA: $P<0.005$ ). When lines were compared within each block, fat body transcript abundance was significantly higher in the high- vs. low-activity lines of Bk-3 $(P<0.005)$, but not in Bk-1 $(P=0.2)$, although rank-order differences between lines were the same for each block. Transcript abundance was also significantly higher in Bk-1 vs. Bk-3 $(P<0.05)$. However, no Line $\times$ Block interaction was observed $(P=0.17)$ indicating that the relative elevation in transcript abundance in high- vs. low-activity lines did not differ significantly between blocks.

The transcript abundance in midgut, measured on day 4 of the last nymphal stadium, was 2.5-fold higher in lines selected for high- vs. low-plasma JHE activity $(P<0.005$; activities pooled across the two blocks). Transcript abundance was higher in high vs. low lines within each block (ANOVA, Bk-1: $P<0.025$; BK3: $P<0.025)$. Finally, as was the case for fat body, Jhe transcript abundance was significantly higher in Bk-1 vs. Bk-3 $(P<0.005)$, and no Line $\times$ Block interaction was observed $(P>0.6)$.

In fat body or midgut, on day 4 of the last nymphal stadium, Jhe transcript abundance was positively correlated with JHE enzyme specific activity of the same organ, or with plasma JHE activity (Table 2). Jhe transcript abundance in fat body was also positively correlated with transcript abundance in midgut (Table 2).

\section{Discussion}

Characterizing genetically-based endocrine variation within populations of organisms is one of the most important tasks facing the nascent field of evolutionary endocrinology (Zera et al., 2007).
Information on the types of endocrine variation available for selection to act on, the extent to which endocrine variants are genetically correlated with each other and hence are constrained to evolve in concert, and the effects of endocrine variants on whole organism traits, are key pieces of information for identifying the mechanisms underlying microevolutionary change in endocrine regulation.

In the present study we measured developmental profiles of JHE and JHEH activities in plasma, fat body and midgut, and Jhe transcript abundance in fat body and midgut in stocks of G. assimilis that differ dramatically in plasma JHE activity due to artificial selection. The main purpose was to identify significant contributors to genetic variation in plasma JHE activity as well as to identify traits that potentially interact genetically (i.e., are correlated) with each other (e.g. JHE and JHEH activities, Jhe transcript abundance in midgut and fat body). On the one hand, because JHE and JHEH both metabolize the same substrate one might expect to find correlations between organ/tissue-specific activities of these enzymes that co-ordinate metabolism of JH within or between various organs. On the other hand, lack of correlations between JHE and JHEH activities could also be important in the tissue-specific regulation of JH levels. To our knowledge, these data constitute the first published data on population-genetic variation in either JHE activity in non-hemolymph body compartments or in Jhe transcript abundance in insects. In addition, JHE and JHEH fat body and midgut enzyme activities reported here are the first reports of activities of these important $\mathrm{JH}$ regulators in individual, non-hemolymph organs of a hemimetabolous insect, a member of a major group of insects that has been understudied with respect to The regulation. Functional aspects of organ-specific JHE and JHEH activities and Jhe transcript abundance will be discussed first, followed by evolutionary aspects.

\subsection{Fat body and midgut JHE and JHEH activities: developmental and functional aspects}

A major finding of the present study was the dramatic difference in fat body JHE activity between lines artificially selected for high- or low-plasma JHE activity (Figure 1). Line differences in fat body JHE activity, strongly paralleled line differences in plasma JHE activity, while developmental changes in fat body JHE activity within individual lines also strongly covaried with plasma activity (Figure 1, Table 1). An important exception to this pattern occurred in the latter part of the last stadium, when fat body JHE activities remained high after plasma JHE activities had dropped precipitously (Figure 1; discussed below). These results are similar to those reported for lepidopterans where high JHE activity occurs in fat body, and where fat body and plasma JHE activity developmental profiles were, in general, strongly correlated. This was especially the case for Trichoplusia ni (Wing et al., 1981; Hammock, 1985), and to a lesser degree for M. sexta (Jesudason et al., 1992). In contrast to the single JHE plasma activity peak during development in hemimetabolous insects such as G. assimilis, holometabolous insects have two plasma JHE activity peaks. In T. ni, JHE activity changed largely in concert in both fat body and plasma during each of these peaks, the exception being the retention of high fat body JHE activity, when plasma JHE activity had dropped after the second peak; by contrast, in $M$. sexta, there was only a single peak in fat body JHE activity that strongly paralleled the first plasma JHE activity peak (Jesudason et al., 1992).

As mentioned previously, plasma JHE activity is produced in non-hemolymph tissues and is secreted into the hemolymph. Several pieces of evidence collectively suggest that fat body JHE, as opposed to midgut JHE, is a greater contributor to the hemolymph JHE activity pool in G. assimilis. Developmental changes 
in JHE specific activity in fat body more strongly paralleled changes in plasma JHE activity than did midgut activity (Figure 1), and fat body JHE activity was more strongly correlated with plasma JHE activity (Table 1). JHE specific activity in the high-activity lines also was about twice as high in the fat body as in the midgut within the same lines.

A potentially important factor that influences the relative contribution of JHE from various organs to the hemolymph JHE pool, but which has not been extensively studied, is the relative secretion rates of JHE from these organs. Estimates of the contribution of JHE from various organs to the plasma JHE activity pool based on the relative magnitude of JHE activity and the masses of these non-hemolymph organs could give erroneous estimates if the rate of JHE secretion into the hemolymph differs significantly between the organs. We have no data on relative JHE secretion rates from various organs in G. assimilis. Although in vitro secretion of JHE from the fat body has been reported for several lepidopterans (Hammock, 1985; Jones et al., 1987) relative secretion rates of JHE from various organs has not been determined. As mentioned above, in both G. assimilis (high-activity lines; Figure 1) and T. ni (Wing et al., 1981; Figure 3) fat body JHE activity remained elevated during the latter part of the last juvenile stadium, when JHE activity in the plasma of both of these species strongly dropped. This suggests that developmental changes in the rates of JHE secretion from fat body may significantly influence the developmental profile of plasma JHE activity.

The non-parallel developmental profiles of plasma, midgut, and fat body JHE activities, during specific periods of the last juvenile stadium of $G$. assimilis also suggest that $\mathrm{JH}$ degradation may differ among various organs, genetic stocks, and stages of development in G. assimilis as has been proposed for a number of lepidopterans (Hammock, 1985; Jesudason et al., 1992). Degradation of JH by JHE in tissues other than hemolymph has not been well studied in insects but is likely to be an important component of $\mathrm{JH}$ titer regulation, especially when the JH titer within specific tissues must differ from that of the hemolymph. For example, JHE activity within imaginal disks of Galleria mellonella peaks before plasma JHE activity and may regulate imaginal disk cell proliferation by reducing the JH concentration within the disks (Reddy et al., 1980).

In general, fat body and midgut $\mathrm{JH}$-epoxide hydrolase activities differed to a much lesser degree between lines, which is consistent with the previous observation of no line differences in JHEH activities in whole-body homogenates (Zera and Zhang, 1995; Zera and Huang, 1999; Zera, 2006). The main exception was midgut JHEH in Bk-3, where activity was higher in the high- vs. low-selected lines throughout the mid-stadium. Specific activity of JHEH was about 2-3-fold higher than JHE in the midgut in both blocks, indicating that JHEH may play a more significant role in $\mathrm{JH}$ degradation in midgut than in fat body.

\subsection{JHE transcript abundance}

Several studies of holometabolous insects have demonstrated a strong correlation between developmental changes in The transcript abundance in fat body and JHE enzyme activity in fat body, plasma, or whole-body extracts (Hirai et al., 2002; Bai et al., 2007; Munyiri and Ishikawa, 2007). These correlations suggest that developmental changes in Jhe gene transcription may be a common cause of developmental changes in JHE specific activity. The present study is the first to report analogous genetic differences in Jhe transcript abundance and genetic covariance between Jhe transcript abundance and JHE enzyme activity in fat body, midgut or plasma at the same time in development in genetic stocks derived from natural populations (Figure 1, Figure 2, and Figure 3; Table 2).

\subsection{Evolutionary implications of tissue and organ specific genetic variation for JHE activity and Jhe gene expression}

The present study essentially measured correlated (indirect) responses to direct artificial selection on plasma JHE activity (Zera and Zhang, 1995; Zera, 2006). Correlated responses to selection identify traits that are genetically correlated due two very different causes (Garland and Carter, 1994). The first occurs when traits are mechanistically linked in a chain of causality determining the expression of a particular trait, such as when a specific DNA sequence specifies a high-activity enzyme phenotype; the DNA sequence will increase in frequency and mean enzyme activity will increase in a line selected for high-enzyme activity. The second type results when different traits interact genetically, for example, when they are regulated by the same genes (i.e. pleiotropy).

Functional considerations discussed above implicate fat body Jhe transcript abundance and fat body JHE enzyme specific activities as important contributors to plasma JHE activity. This information, coupled with the correlated responses in fat body Jhe transcript abundance and fat body JHE specific activity (both elevated in lines selected for high plasma JHE activity), identify these two traits as being important contributors to genetic variation in plasma JHE activity, the trait that was directly selected. The cause of the correlated response to selection on midgut JHE activity is less certain, because the extent to which midgut JHE activity contributes to plasma JHE activity is less clear. Thus, midgut Jhe transcript abundance and midgut JHE specific activity may have been indirectly altered by selection either due to the contribution of midgut JHE to plasma JHE, or due to midgut Jhe and fat body the being co-regulated by the same factors. Additional functional and genetic studies will be necessary to distinguish between these two hypotheses.

Because selection was initiated on a laboratory base population, that had been kept for only a few generations after being founded from field-collected individuals (Zera and Zhang, 1995), genetic variation for fat body and midgut Jhe transcript abundance and JHE enzyme specific activity were likely present in the field populations from which founding crickets were collected. These findings are significant as there is a paucity of information on the characteristics of standing genetic variation for endocrine traits in either laboratory or field populations. This is especially the case for molecular-genetic aspects of endocrine variation such as variation in Jhe transcript abundance. Most current examples of molecular-genetic variation for endocrine traits in natural populations are for traits documented in vertebrates (Zera et al., 2007).

Results of the present study, which directly identified genetic covariation (i.e. correlated responses to selection) between The gene expression, JHE activity in non-hemolymph tissues, and plasma JHE activity, provide support for earlier arguments that genetic variation in plasma JHE activity in Gryllus is more likely due to variation in Jhe gene expression rather than to differences in kinetic properties of stock-specific JHE enzyme(s) (Zera and Huang, 1999). No substantial kinetic differences have been identified between JHE enzymes from high- or low-activity selected lines of G. assimilis (Zera and Zeisset, 1996; Table 3), or its congener G. rubens (Zera et al., 1992). Nor have any nucleotide differences been identified in the coding sequence of a Jhe cDNA of $G$. assimilis within or between the same lines that were the subjects of the present study (Crone et al., 2007; Table 3).

At present, relatively few population-genetic studies have investigated correlations between gene expression and enzyme specific activity, and for those that have, no consensus has emerged regarding the extent to which strong correlations exist between these two factors. For example, at one extreme, studies in the kil- 
Table 3. Summary of correlated responses to selection (i.e. line differences) in lines of G. assimilis artificially selected for high- or low-plasma JHE activity on day 3 of the last nymphal stadium

\begin{tabular}{|c|c|c|}
\hline Trait & Value in lines selected for high- vs. low-plasma JHE activity & References \\
\hline (1) Plasma JHE activity (mid-last stadium) & Significantly higher (6-8 fold) & $A, B, D, E, G$ \\
\hline (2) Plasma JHE activity in adults & Marginally higher & $\mathrm{A}, \mathrm{B}, \mathrm{D}, \mathrm{E}$ \\
\hline (3) Whole-body JHE activity & 2.6-Fold higher & $\mathrm{A}, \mathrm{E}$ \\
\hline (5) JH binding in plasma & 3-Fold higher & $\mathrm{B}, \mathrm{E}$ \\
\hline (6) JHEH whole specific body activity & No difference & A, E \\
\hline (7) Whole-body, in vivo rate of JH degradation & 1.4-Fold higher & A, B \\
\hline (8) JHE enzyme kinetics & No difference in Michaelis constant, inhibition constants or thermostability & $\mathrm{C}$ \\
\hline (11) JHE specific activity in midgut & 1-Fold higher at day 5 peak; not different on most days & G \\
\hline (12) Jhe transcript abundance in fat body & 1.9-Fold higher & G \\
\hline (13) Jhe transcript abundance in midgut & 2.5-Fold higher & G \\
\hline (14) JHEH activity in fat body & No consistent difference & G \\
\hline (15) JHEH activity in midgut & No consistent difference & G \\
\hline
\end{tabular}

All traits were measured in the last nymphal stadium except for plasma JHE activity in adults. References: A: Zera and Zhang, 1995; B: Zera et al., 1996; C: Zera and Zeisset, 1996; D: Zera et al., 1998; E: Zera and Huang, 1999; F: Crone et al., 2007; G: present study. See Zera and Huang (1999) and Zera (2006) for additional information regarding comparisons between long-wing and short-wing selected lines of G. firmus and G. rubens for many of these correlated responses.

lifish Fundulus heteroclitus, have demonstrated that genetic differences in lactate dehydrogenase (LDH) transcript abundance explain a large proportion of genetic variation in $\mathrm{LDH}$ specific activity found in natural populations of this species (Crawford and Powers, 1989). At the other extreme, no difference in alcohol dehydrogenase $(A d h)$ transcript abundance was identified between genetic stocks of Drosophila melanogaster that differ in specific activity of the ADH enzyme, as well as in ADH enzyme protein concentration (Laurie and Stam, 1988). Finally in laboratory selection studies of microorganisms, microevolutionary changes in enzyme activities often are not accompanied by changes in transcript abundance and vice versa (e.g. Daran-Lapujade et al., 2004; and references therein).

In the present study we found no correlated responses to selection on midgut or fat body JHEH specific activity when plasma JHE activity was directly selected (Table 3). Thus, alleles that influence fat body and midgut JHE specific activity and, in turn, plasma JHE activity have no observable effect on fat body or midgut JHEH activity. The failure to find correlated responses to selection on either fat body or midgut JHEH specific activity, is consistent with our previous finding of no correlated responses to selection on whole-body JHEH activity (Zera and Huang, 1999; Zera, 2006). These results stand in stark contrast with the strong correlated responses to selection and strong positive genetic correlations between plasma JHE activity and plasma JH binding (see Discussion in Zera et al., 1996; Zera, 2006; Table 3). From an evolutionary perspective, the significance of these findings is that activities of the two main JH degrading enzymes, JHE and JHEH, can evolve independently of each other, while plasma $\mathrm{JH}$ binding and $\mathrm{JH}$ degradation are constrained to evolve in concert, possibly because they are co-regulated by the same factors. At present it is unclear why certain components of $\mathrm{JH}$ regulation are genetically correlated while others are not.

Plasma JHE activity is a complex trait, and there are still many potential contributors to genetic differences in this trait between selected lines of G. assimilis that have yet to be investigated. Two especially important foci for future research are rate of secretion of JHE into the hemolymph and the role of variation in expression of additional Jhe genes. Previous studies have demonstrated that high-and low-activity lines of several Gryllus species differ dramatically in the proportion of whole-body JHE activity that is found in the plasma vs. other body compartments (Table 3). This suggests that (1) genetic variation in JHE secretion into the hemolymph is a likely important contributor to genetic variation in plasma JHE ac- tivity in G. assimilis, and that (2) natural selection on genes regulating secretion rates has been an important aspect of the evolution of high- and low-JHE activity of genetically-specified morphs of G. firmus that express different wing-length and life-history traits. Stock-specific rates of JHE secretion into the hemolymph have yet to be directly studied in any Gryllus species. Finally, multiple JHE isoforms in G. assimilis have been identified that differ in up to 4 residues of the 13-20 N-terminal amino-acid sequences of the mature proteins (Zera et al., 2002; Crone et al., 2007). This suggests the possible existence of multiple Jhe genes that encode multiple JHE enzymes in this species. The contribution of differential expression of additional Jhe genes to genetic variation in plasma JHE enzyme activity has not yet been investigated.

In summary, we have identified genetic variation in several traits, most notably JHE specific activity in fat body, and The gene transcript abundance, that contribute to genetic variation in plasma JHE activity in G. assimilis. These findings represent significant contributions to our understanding of the chain of causality the leads from alteration in regulation of Jhe gene(s) to alteration in plasma JHE activity for this important experimental model in evolutionary endocrinology.

\section{Acknowledgement}

Research reported in this manuscript was supported by NSF grant IBN-0212486 to A. J. Zera.

\section{References}

Bai et al., 2007 - H. Bai, P. Ramaseshadri and S. R. Palli, Identification and characterization of juvenile hormone esterase gene from the yellow fever mosquito, Aedes aegypti, Insect Biochemistry and Molecular Biology 37 (2007), pp. 829-837.

Crawford and Powers, 1989 - D. L. Crawford and D. Powers, Molecular basis of evolutionary adaptation at the lactate dehydrogenase-B locus in the fish Fundulus heteroclitus, Proceedings of the National Academy of Sciences United States of America 86 (1989), pp. 9365-9369.

Crone et al., 2007 - E. Crone, A. J. Zera, A. Anand, J. Oakeshott, T. Sutherland, R. Russell, L. G. Harshman, F. Hoffman and C. Claudianos, Jhe in Gryllus assimilis: cloning, sequence-activity associations and phylogeny, Insect Biochemistry and Molecular Biology 37 (2007), pp. 1359-1365.

Daran-Lapujade et al., 2004 - P. Daran-Lapujade, M. Jansen, J. M. Daran, W. Van Gulik, J. H. de Winde and J. T. Pronk, Role of transcriptional regulation in controlling fluxes in central carbon metab- 
olism of Saccharomyces cerevisiae: a chemostat culture study, Journal of Biological Chemistry 279 (2004), pp. 9125-9138.

Garland and Carter, 1994 - T. Garland Jr. and P. Carter, Evolutionary physiology, Annual Review of Ecology and Systematics 56 (1994), pp. 579-621.

Goodman and Granger, 2005 - W. G. Goodman and N. A. Granger, The juvenile hormones. In: L. I. Gilbert, K. Iatrou and S. S. Gill, Editors, Comprehensive Molecular Insect Science vol. 3, Elsevier, Amsterdam (2005), pp. 319-406.

Hammock and Sparks, 1977 - B. D. Hammock and T. C. Sparks, A rapid assay for juvenile hormone esterase activity, Analytical Biochemistry 82 (1977), pp. 573-579.

Hammock, 1985 - B. D. Hammock, Regulation of the juvenile hormone titer: Degradation. In: G. A. Kerkut and L. I. Gilbert, Editors, Comprehensive Insect Physiology, Biochemistry, and Pharmacology vol 7, Oxford, Pergamon (1985), pp. 431-472.

Hirai et al., 2002 - M. Hirai, M. Kamimura, K. Kikuchi, Y. Yasukochi, M. Kiuchi, T. Shinoda and T. Shiotsuki, cDNA cloning and characterization of Bombyx mori juvenile hormone esterase: an inducible gene by the imidizole insect growth regulator KK-42, Insect Biochemistry and Molecular Biology 32 (2002), pp. 627-635.

Jesudason et al., 1992 - P. Jesudason, D. D. Anspaugh and R. M. Roe, Juvenile hormone metabolism in the plasma, integument, midgut, fat body, and brain during the last instar of the tobacco hornworm, Manduca sexta, Archives of Insect Biochemistry and Physiology 20 (1992), pp. 87-105.

Jones et al., 1987 - G. Jones, D. Jones and S. Hiremeth, An in vitro system for studying juvenile hormone induction of juvenile hormone esterase from the fat body of Tricoplusia ni, Insect Biochemistry $\mathbf{1 7}$ (1987), pp. 897-904.

Laurie and Stam, 1988 - C. C. Laurie and L. F. Stam, Quantitative analysis of RNA produced by slow and fast alleles of Adh in Drosophila melanogaster, Proceedings of the National Academy of Sciences United States of America 85 (1988), pp. 5161-5165.

Munyiri and Ishikawa, 2007 - F. N. Munyiri and Y. Ishikawa, Molecular cloning and developmental expression of the gene encoding juvenile hormone esterase in the yellow-spotted longicorn beetle, Psacothea hilaris, Insect Biochemistry and Molecular Biology 37 (2007), pp. 497-505.

Reddy et al., 1980 - G. Reddy, D. C. McCaleb and A. K. Kumaran, Tissue distribution of juvenile hormone hydrolytic activity in Galleria mellonella larvae, Experientia 36 (1980), pp. 461-462.

Roe and Venkatesh, 1990 - R. M. Roe and K. Venkatesh, Metabolism of juvenile hormones: degradation and titer regulation. In: A. P. Gupta, Editor, Morphogenetic Hormones of Arthropods vol. 1, Rutgers University Press, New Brunswick (1990), pp. 126-179.

Roff et al., 1997 - D. Roff, G. Stirling and D. J. Fairbairn, The evolution of threshold traits: a quantitative genetic analysis of the physiological and life-history correlates of wing dimorphism in the sand cricket, Evolution 51 (1997), pp. 1910-1919.

Share and Roe, 1988 - M. R. Share and R. M. Roe, A partition assay for simultaneous determination of insect juvenile hormone esterase and epoxide hydrolase activity, Analytical Biochemistry 169 (1988), pp. 81-88.
Sokal and Rohlf, 1989 - R. R. Sokal and F. J. Rohlf, Biometry (2nd ed.), W. H. Freeman, San Fancisco (1989).

Sokal and Rohlf, 1969 - R. R. Sokal and F. J. Rohlf, Statistical Tables, W. H. Freeman, San Francisco (1969).

Wing et al., 1981 - K. D. Wing, T. C. Sparks, V. M. Lovell, S. O. Levinson and B. D. Hammock, The distribution of juvenile hormone esterase and its interrelationship with other proteins influencing juvenile hormone metabolism in the cabbage looper, Tricoplusia ni, Journal of Insect Physiology 11 (1981), pp. 473-485.

Zera, 2006 - A. J. Zera, Evolutionary genetics of juvenile hormone and ecdysteroid regulation in Gryllus: a case study in the microevolution of endocrine regulation, Comparative Biochemistry and Physiology, Part A 144 (2006), pp. 365-379.

Zera, 2004 - A. J. Zera, The endocrine regulation of wing polymorphism: State of the art, recent surprises, and future directions, Integrative and Comparative Biology 43 (2004), pp. 607-616.

Zera et al., 1992 - A. J. Zera, X. Gu and M. Zeisset, Characterization of juvenile hormone esterase from genetically-determined wing morphs of the cricket, Gryllus rubens, Insect Biochemistry and Molecular Biology 22 (1992), pp. 829-839.

Zera et al., 2007 - A. J. Zera, L. G. Harshman and T. D. Williams, Evolutionary endocrinology: the developing synthesis between endocrinology and evolutionary genetics, Annual Review of Ecology, Evolution and Systematics 38 (2007), pp. 793-817.

Zera and Huang, 1999 - A. J. Zera and Y. Huang, Evolutionary endocrinology of juvenile hormone esterase: Functional relationship with wing polymorphism in the cricket, Gryllus firmus, Evolution 53 (1999), pp. 837-847.

Zera et al., 2002 - A. J. Zera, T. Sanger, J. Hanes and L. Harshman, Purification and characterization of hemolymph juvenile hormone esterase from the cricket, Gryllus assimilis, Archives of Insect Biochemistry and Physiology 49 (2002), pp. 41-55.

Zera et al., 1998 - A. J. Zera, T. Sanger and G. L. Cisper, Direct and correlated responses to selection on JHE activity in adult and juvenile Gryllus assimilis: implications for stage-specific evolution of insect endocrine traits, Heredity 80 (1998), pp. 300-309.

Zera et al., 1996 - A. J. Zera, J. Sall and R. Schwartz, Artificial selection on JHE activity in Gryllus assimilis: nature of activity differences between lines and effect on JH binding and metabolism, Archives of Insect Biochemistry and Physiology 32 (1996), pp. 421-428.

Zera and Tiebel, 1989 - A. J. Zera and K. C. Tiebel, Differences in juvenile hormone esterase activity between presumptive macropterous and brachypterous Gryllus rubens: implications for the hormonal control of wing polymorphism, Journal of Insect Physiology 35 (1989), pp. 7-17.

Zera and Zeisset, 1996 - A. J. Zera and M. Zeisset, Biochemical characterization of juvenile hormone esterases from lines selected for high or low enzyme activity in Gryllus assimilis, Biochemical Genetics 34 (1996), pp. 421-435.

Zera and Zhang, 1995 - A. J. Zera and C. Zhang, Direct and correlated responses to selection on hemolymph juvenile hormone esterase activity in Gryllus assimilis, Genetics 141 (1995), pp. 1125-1134. 